\title{
Immune responses against norovirus GII.4 virus-like particles in mice
}

\author{
H. USHIJIMA ${ }^{1}$, S. MACHIDA ${ }^{2}$, A. NOMURA ${ }^{3}$, P. KHAMRIN ${ }^{4}$, D. N. TRAN ${ }^{1}$, H. NOMURA ${ }^{3}$, S. HAYAKAWA ${ }^{1}$
}

\begin{abstract}
${ }^{1}$ Division of Microbiology, Department of Pathology and Microbiology, Nihon University School of Medicine, Itabashi, Tokyo, Japan; ${ }^{2}$ Medical Research Center, Saitama Medical University, Moroyama, Saitama, Japan; ${ }^{3}$ ImmumoProbe Co., Ranzan, Saitama, Japan; ${ }^{4}$ Department of Microbiology, Faculty of Medicine, Chiang Mai University, Chiang Mai, Thailand
\end{abstract}

Received February 3, 2016; accepted April 1, 2016

\begin{abstract}
Summary. - Norovirus (NoV) is a causative agent of gastroenteritis in children and adults worldwide. To evaluate the safety and effectiveness of a NoV vaccine candidate, $100 \mu \mathrm{g}$ of GII.4 NoV-like particles (VLPs) was challenged orally (oral and intrabuccal administration) and by subcutaneous injection without adjuvant in mice. The subcutaneous injection induced IgG in sera, but not IgA in feces. The oral delivery method induced IgA in both sera and feces, but not IgG in sera. However, challenging by the intrabuccal administration induced IgG in sera and IgA in both sera and feces, especially by 2 -dose immunization. The peak of specific immune responses by the intrabuccal administration was detected later than that of the oral delivery method. Heterologous immune responses against other genotypes were also recognized. NoV-specific IFN- $\gamma$ was detected after the intrabuccal administration. These findings indicated that the administration of NoV VLPs by intrabuccal administration could induce the best immune responses against NoV in mice.
\end{abstract}

Keywords: norovirus; virus-like particles (VLPs); mucosal immunity

\section{Introduction}

Norovirus (NoV) is one of the most common pathogens that is associated with acute gastroenteritis (Glass et al., 2009). Symptoms caused by NoV are usually mild and self-limiting. However, NoV is highly contagious and easily transmitted from person to person in hospitals, schools, and restaurants (Glass et al., 2000). NoV is sometimes able to cause severe illnesses, especially in young children, elderly and immunocompromised patients (Bok and Green, 2012). NoVs belong to the family Caliciviridae and contain a singlestranded positive-sense RNA genome. Genome has 3 open reading frames (ORFs); ORF1 encoding the nonstructural proteins, ORF2 encoding major capsid protein (VP1), and ORF3 encoding minor capsid protein (VP2) (Prasad et al., 1999). NoV is divided into five genogroups (GI-GV). Of

E-mail: ushijima-hiroshi@jcom.home.ne.jp; phone: +81339728111.

Abbreviations: dpi $=$ days post infection; $\mathrm{NoV}=$ norovirus; rNoV = recombinant NoV; $\operatorname{VLP}(\mathrm{s})=$ virus-like particle $(\mathrm{s})$ these, NoV GI and GII are commonly found in humans, and are further subdivided into 9 and 22 different genotypes, respectively (Kroneman et al., 2013). NoV GII.4 is responsible for more than $70 \%$ of sporadic (Fankhauser et al., 2002) and epidemic NoV-associated outbreaks (Lindesmith et al., 2012).

NoVs cannot grow in cell culture, but the expression of VP1 in insect cells results in the formation of virus-like particles (VLPs) that are morphologically and antigenically similar to the native NoV (Okamae et al., 2007; Jiang et al., 1992). Using human recombinant NoV (rNoV) VLPs, serological studies showed a high prevalence of NoV-specific IgG and IgA in human sera (Gray et al., 1994; Parker et al., 1995). Other studies reported the development of cross-reactive antibodies after patients were infected with a specific NoV type (Rockx et al., 2005; Parker et al., 1994). The mucosal immunization of rNoV VLP induced systemic and mucosal immune responses in mice (Ball et al., 1998; Guerrero et al., 2001). IgA against rNoV VLPs increased by the oral administration with or without cholera toxin (Periwal et al., 2003). The clinical trial using rNoV GI.1 VLPs as a vaccine candidate had been examined in humans. The results 
indicated that the specific IgAs were induced in sera, and gastroenteritis was reduced to $37 \%$ after administration of an intranasal rNoV GI.1 VLPs-based vaccine with adjuvant (Atmar et al., 2011).

For the safety and potential induction of mucosal immunities, we tested the level of serum IgG, IgA, and intestinal IgA in mice after oral immunizations (absorbed administration and oral delivery) and subcutaneous immunization by using rNoV GII.4 VLPs without external adjuvants.

\section{Materials and Methods}

Recombinant NoV (rNoV) VLPs preparation and characterization. NoV GI.1, GI.11, GII.2, GII3, GII.4 and GII.6 were detected in feces of Japanese patients with gastroenteritis. NoV RNA was extracted, and the entire NoV capsid gene was amplified by RTPCR with primers Mex20attB1S and attB2TX30SXN (Jiang et al., 1992). The amplicons were sequenced and compared with other NoV reference strains available in EMBL/GenBank database. The recombinant plasmids containing capsid gene were constructed by inserting the NoV capsid sequence to pDONR221 and pDEST-8 vectors, and then transformed into $\mathrm{DH} 10 \mathrm{Bac}^{\mathrm{TM}}$ competent Escherichia coli by Bac-to-Bac Baculovirus expression system (Invitrogen, Life Technologies, Japan), according to the manufacturer's instructions. The Spodoptera frugiperda 9 (Sf9) insect cell lines were transfected by recombinant bacmid, and then cultured in TC100 medium at $27^{\circ} \mathrm{C}$. After $4-5$ days post infection (dpi), the culture supernatant was harvested and used as a seed baculovirus. The Tn 5 cells were infected with the seed baculovirus at $27^{\circ} \mathrm{C}$, and harvested after $6 \mathrm{dpi}$. The secreted VLPs in cell culture medium were separated by ultracentrifugation and the obtained viral pellet was resuspended in PBS $\mathrm{pH}$ 7.2. The VLPs were purified by a $\mathrm{CsCl}$ equilibrium gradient ultracentrifugation. Purified VLPs were analyzed by SDS-PAGE to confirm their protein composition, and then examined by negative-staining electron microscopy. Protein concentration was determined by a commercial Bradford assay kit (Thermo Fisher Scientific Inc., USA). Antigenicity of rNoV VLP preparations were confirmed by Western blot analysis using hyperimmune antisera against NoV.

Immunization and sample collection. (1) Immunization. Female 5-week-old BALB/c mice were obtained from Saitama Experimental Animal Supply Co. Ltd (Saitama, Japan). Mice were allowed to acclimate for 1 week under constant conditions. This study was carried out according to the guideline for Animal Study, and all animal experiments were approved by the Institutional Animal Care and Use, Japan (permission No. H24-001 IP). Mice were immunized subcutaneously or orally with NoV GII.4 VLPs in PBS on day 0 . For oral immunization, VLPs were challenged with a gastric gavage using a stainless steel incubation needle or by an intrabuccal administration using a dropper. One hundred $\mu \mathrm{g}$ of VLPs were administered to mice once without adjuvant. For a booster challenge, mice were re-inoculated on day 21. Mice receiving only PBS were used as controls. (2) Sample collection. Blood samples were collected by tail bleed. Fecal samples were obtained from individual mice by a fecal collection cage, and resuspended in 5\% w/v of $25 \%$ Immuno Block (DS Pharma Biomedical Co., Japan) with PBS-T. Each suspension was thoroughly vortexed, and centrifuged for 10 $\min$ at $18,000 \mathrm{xg}$. The supernatant was collected, and fetal bovine serum (FBS) was added to make the final concentration of $10 \%$. The samples were stored at $-80^{\circ} \mathrm{C}$ until tested. Individual serum and fecal samples were collected before the first immunization (pre-immune samples) for comparison.

Detection of immune response by ELISA. (1) Serum anti-NoV IgG, IgA ELISAs. NoV-specific IgG titers in sera from immunized mice were determined by ELISA as described previously (Tacket et al., 2003). The plates (Maxisorp, Nunc, Denmark) were coated with $1 \mu \mathrm{g}$ rNoV VLPs in carbonate-bicarbonate buffer ( $\mathrm{pH}$ 9.6), and then incubated for $4 \mathrm{hr}$ at RT, then washed 3 times with PBS-T, and blocked with 25\% Immuno Block in PBS containing 0.05\% Tween-20 (PBS-T), and kept overnight at $4^{\circ} \mathrm{C}$. The plates were washed 3 times with PBS-T, and then a 1:500 diluted immunized serum with PBS-T was added to each well, and incubated at $37^{\circ} \mathrm{C}$ for $1 \mathrm{hr}$. After washing, horseradish peroxidase (HRP) conjugated rabbit anti-mouse IgG $(\mathrm{H}+\mathrm{L})$ (Zymax, Invitrogen, Life Technologies Co., Japan) or HRP conjugated goat anti-mouse IgA (Bethyl Laboratories, Inc. USA) was added to each well at a dilution of 1:20,000 and 1:40,000, respectively. The secondary antibody was incubated for $1 \mathrm{hr}$ at $37^{\circ} \mathrm{C}$ and then O-phenylenediamine dihydrochloride substrate (Sigma-Aldrich, Tokyo, Japan) was added. After $30 \mathrm{~min}$, the color reaction was stopped with $2 \mathrm{~mol} / \mathrm{l}$ sulphuric acid $\left(\mathrm{H}_{2} \mathrm{SO}_{4}\right)$. Optical density at the $492 \mathrm{~nm}$ with a reference wavelength of 630 nm was measured (Sjeia Auto Reader II; Sanko Junyaku Co. Ltd., Japan). Background signal (mean OD value from blank wells) was subtracted from all readings on the plates. (2) Fecal anti-NoV IgA ELISA. After the 96-well plates were coated with $\mathrm{rNoV}$ antigen, the fecal supernatants were added to test the NoV specific IgA by ELISA. The plates were incubated for $2 \mathrm{hr}$ at $37^{\circ} \mathrm{C}$. After washing with PBS-T 4 times, the HRP conjugated goat anti-mouse IgA diluted at 1:40,000 in PBS-T containing 25\% Immuno Block ${ }^{\circ}$ was added to each well. The conjugated antibody was incubated for $1 \mathrm{hr}$ at $37^{\circ} \mathrm{C}$. The substrate was added, and $\mathrm{OD}$ was measured.

Interferon gamma ELISPOT assay. Erythrocyte-depleted splenocytes in the immunized mice were assessed by enzyme-linked immunosorbent spot (ELISPOT) assay. The ELISPOT assay was used to measure NoV specific T-cell responses by quantification of IFN- $\gamma$ production from splenocytes by using $\mathrm{BD}^{\mathrm{TM}}$ ELISPOT mouse IFN- $\gamma$ ELISPOT kit (BD Bioscience, USA). One hundred $\mu \mathrm{l}$ of cell suspension with $10^{6} \mathrm{cell} / \mathrm{s} / \mathrm{ml}$ was added to each well. The NoV GII.4 VLPs and a negative control RPMI buffer were added to the well at a concentration of $1 \mu \mathrm{g} / 100 \mu \mathrm{l}$. Assay plates were incubated overnight for $24 \mathrm{hr}$ at $37^{\circ} \mathrm{C}, 5 \% \mathrm{CO}_{2}$ and $95 \%$ humidity. The plates were washed six times with PBS containing bovine serum albumin and $0.005 \%$ Tween-20 (ELISPOT wash buffer). A biotinylated anti-mouse recombinant IFN- $\gamma$ antibody was diluted and $50 \mu \mathrm{l}$ per well were added. After incubation for $2 \mathrm{hr}$ at $37^{\circ} \mathrm{C}$, 
the plates were washed 6 times with ELISPOT wash buffer. The streptavidin-alkaline phosphatase was added, and incubated at RT for $1 \mathrm{hr}$. The plates were washed 3 times with ELISPOT washing buffer, and 3 times with PBS. One hundred $\mu$ l of 1-step nitro blue terazolium-5-bromo-4-chloro-3-indolylphosphate substrate was added to each well, and spots were developed for 5 to $10 \mathrm{~min}$ at (a)

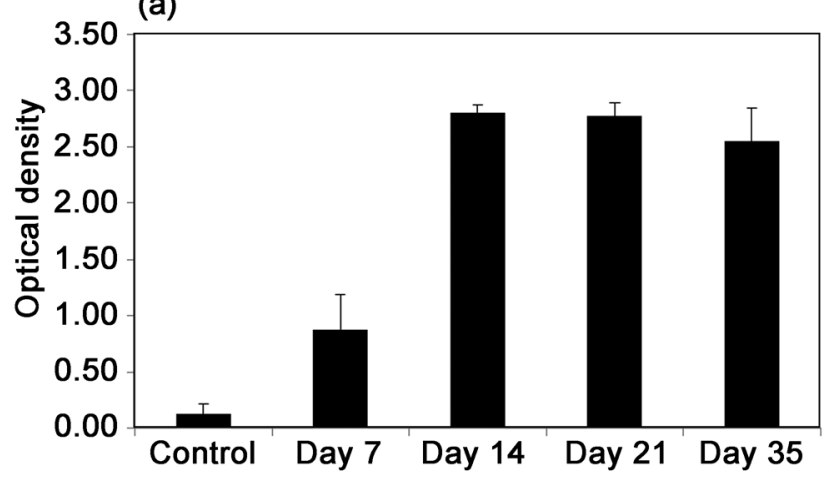

(b)

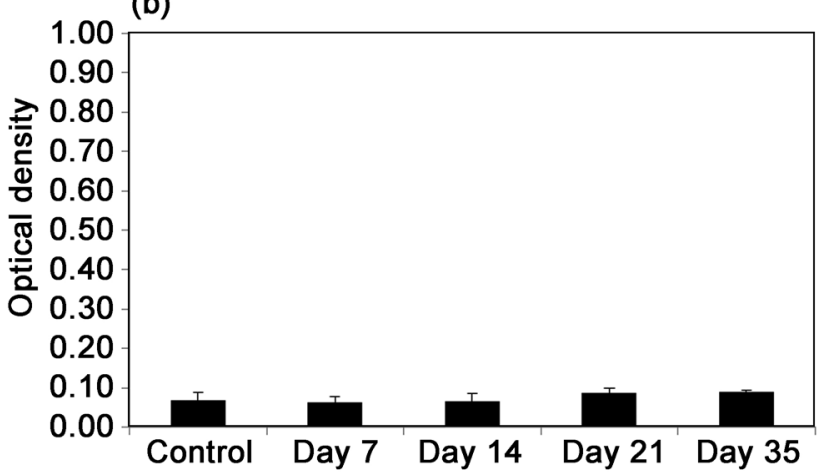

Fig. 1

Immune responses by the subcutaneous injection in mice

Serum IgG (a) and fecal IgA (b) responses after the subcutaneous injection of rNoV GII.4 VLPs. The VLPs were injected via the subcutaneous route at day 0. Serum and fecal samples were collected 7, 14, 21, and 35 dpi. Antibody titers were determined by ELISA.

(a)

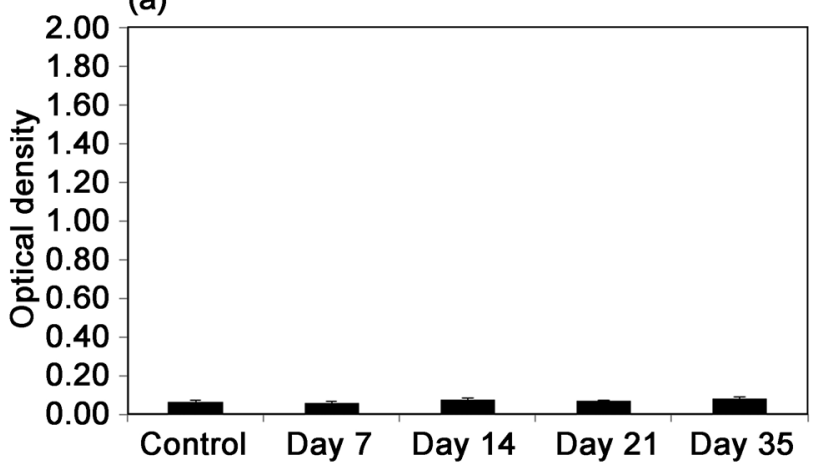

(b)

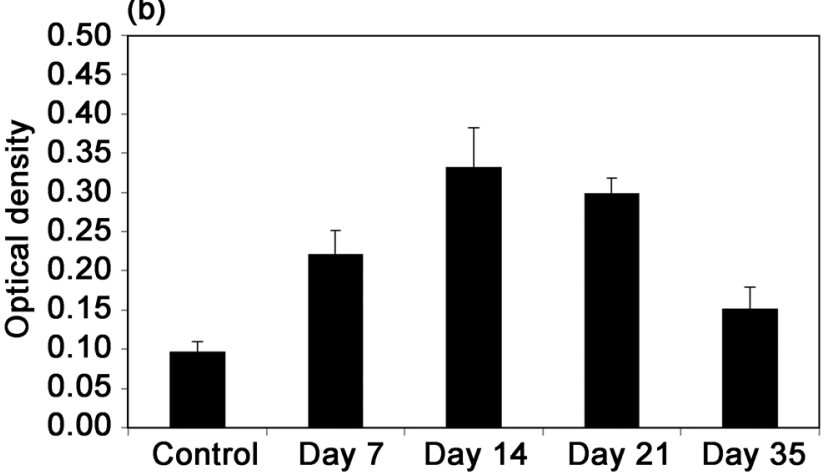

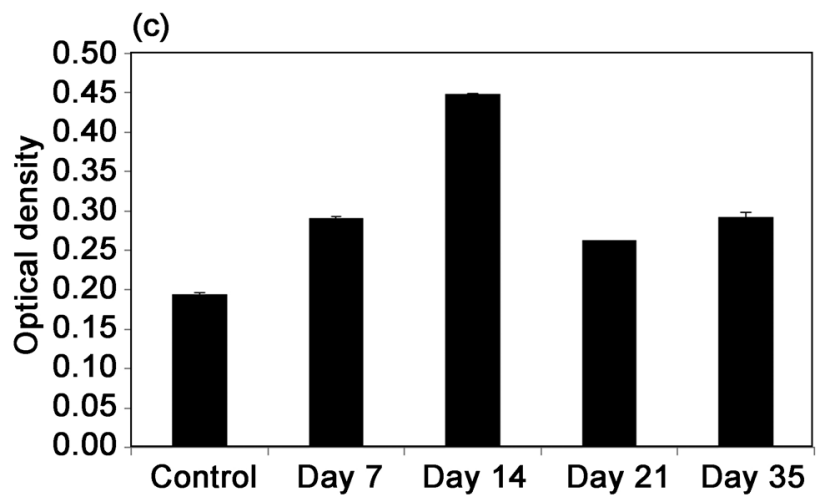

Fig. 2

Immune responses by gavage in mice

The rNoV VLPs were administered by gavage in mice. Serum and fecal samples were collected 7, 14, 21, and 35 dpi. Serum IgG (a), serum IgA (b), and fecal IgA (c) antibody titers were determined by ELISA 
RT. Substrate was discarded and each well was rinsed with water to stop the reaction. The plates were allowed to air dry, and spots were enumerated by counting under a dissecting microscope. To determine the cell specificity, either CD4+ or CD8+ T-cell populations were depleted with magnetic Dynabeads (Veritas, Life Technologies Co.) coated with rat monoclonal antibody against mouse CD4 or CD8. Erythrocyte-depleted mixed cells were incubated with anti CD4 antibodies or anti CD8 antibodies coated magnetic beads on ice, washed, and then passed through a MiniMacs column. Then, the columns were washed with buffer, and unbound cells were collected. The collected cells were resuspended in RPMI buffer, and ELISPOT assays were performed according to the protocol mentioned above. For comparison, CD4+ T cells were also purified using the Miltenyi Biotec CD4+ T-cell isolation kit (Miltenyi Biotec, USA) or the Dynal ${ }^{\mathrm{TM}}$ mouse CD4-negative isolation kit (Dynal Biotech, Invitrogen, Norway) according to the manufacturer's instructions with the minor modifications in order to increase percentage of the purity. The only change from manufacturer's instructions while using the commercial kits was washing the cells with additional buffer after incubation with antibody cocktails and beads.
Statistical analyses. The differences between groups were determined by using one-way analysis of variance (ANOVA). A $p$-value of less than 0.05 (two-tailed) was considered significant.

\section{Results}

Immune responses by the subcutaneous immunization

Mice were immunized subcutaneously with $100 \mu \mathrm{g}$ of rNoV GII.4 VLPs. Their sera and feces were collected at day 7, 14, 21, and 35 after injection. The specific IgG against rNoV GII.4 VLPs in sera had increasing tendency from day 7 as shown in Fig. 1a $(\mathrm{p}<0.05)$. We did not see any increase of the specific IgA in feces (Fig. 1b).

\section{Immune responses by gavage}

The VLP immunization by gavage using the intubation needle did not induce specific IgG response in sera $(p>0.05)$ (Fig. 2a). The specific IgA in both sera and feces was induced (a)

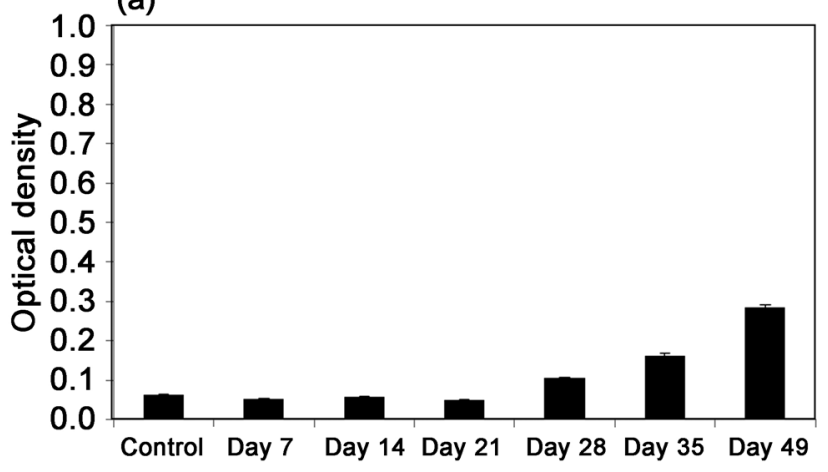

(b)

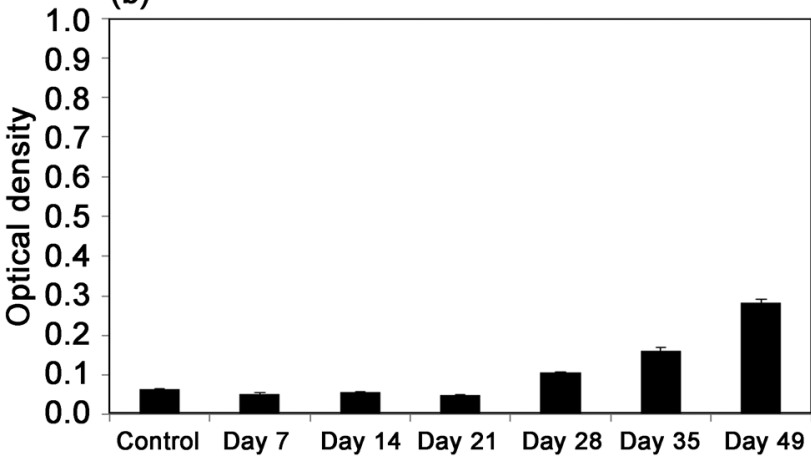

(c)

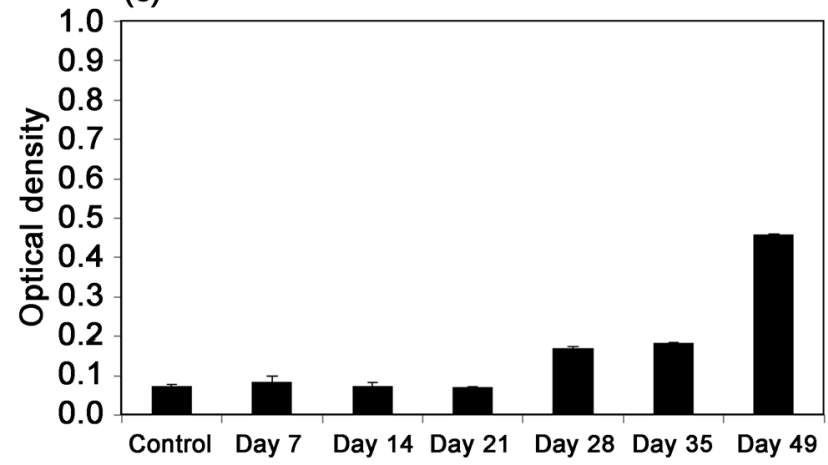

Fig. 3 
(a)

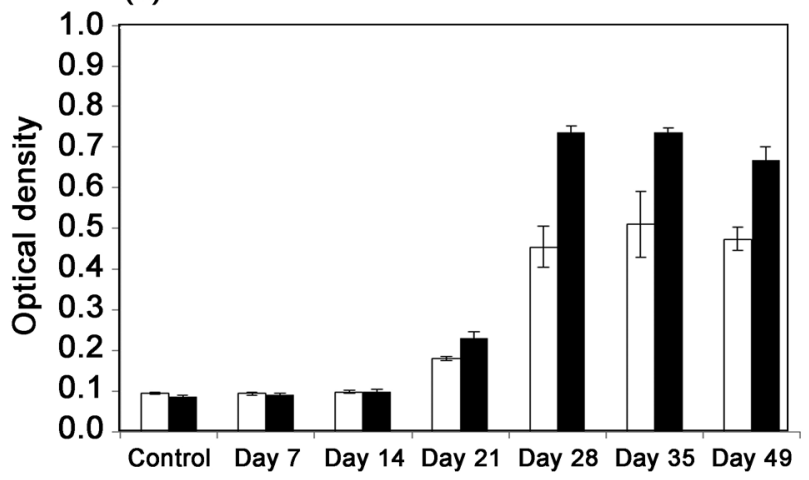

(b)

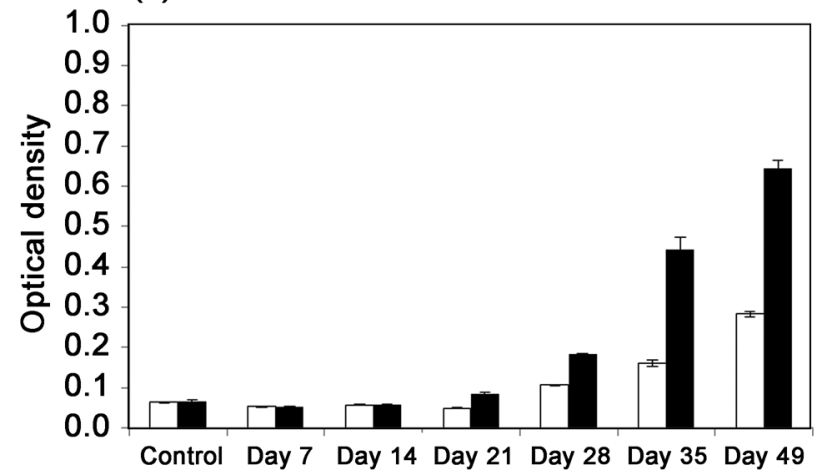

(c)

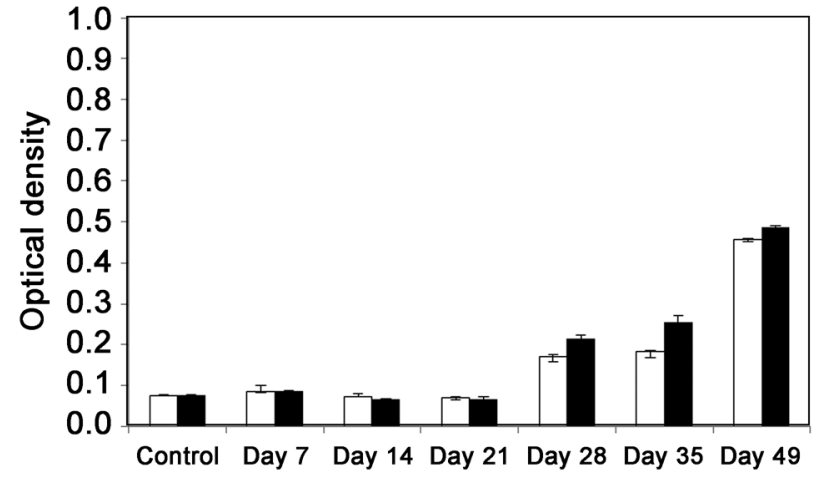

Fig. 4

Boost responses by the intrabuccal administration in mice

The rNoV VLPs were administered to mice by the intrabuccal route on days 0 and 21. 1-dose administration $\square$; 2-dose administration $\mathbf{m}$.

at days 7, 14, 21, and 35 in feces. The highest level of IgA was detected after 14 dpi ( $<$ <.05) (Fig. 2b,c).

Immune responses by the intrabuccal administration

Serum IgG, IgA, and fecal IgA against NoV GII.4 VLPs were measured after the intrabuccal immunization using a dropper. The titers of serum IgG, IgA, and fecal IgA significantly increased from 28 to 49 dpi $(\mathrm{p}<0.05)$ (Fig. 3).

Immune responses for boost challenge by the intrabuccal administration

Immune responses after the 1-dose and 2-dose administrations of NoV VLPs by the intrabuccal administration were examined. Mice were immunized with NoV VLPs twice on days 0 and 21 with $100 \mu \mathrm{g}$ of NoV GII.4 VLPs. The serum IgG and IgA titers were significantly higher in the 2-dose group compared to the 1-dose group. The levels of immune responses significantly increased at 28,35 , and 49 dpi ( $<$ <0.05) (Fig. 4a,b). There were no significant dif-

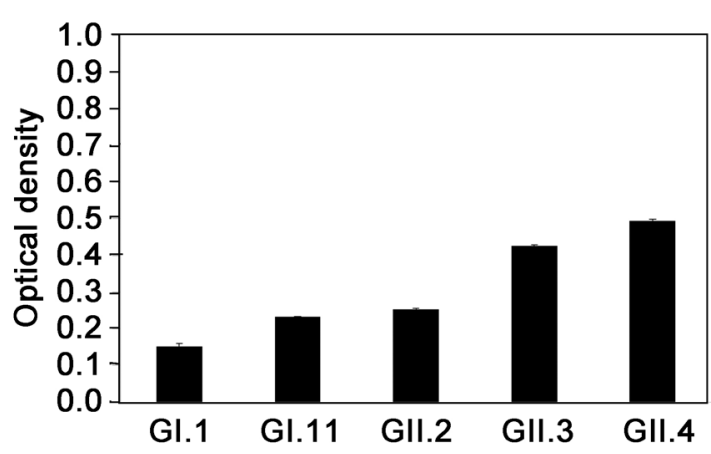

Fig. 5

Cross-reactive fecal IgA to NoV GII.4, GII.3, GII.2, GI.1, GI.11 derived VLPs

Mice were immunized by the intrabuccal administration with rNoV GII.4 VLPs, and fecal IgA were challenged by homologous and heterologous VLPs and evaluated by ELISA.

ferences in the fecal IgA titers between 1-dose and 2-dose groups (Fig. 4c). 
(a)

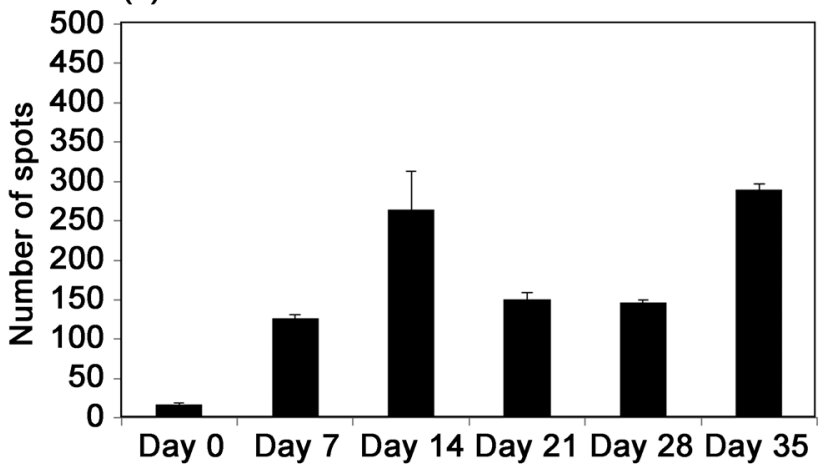

(b)

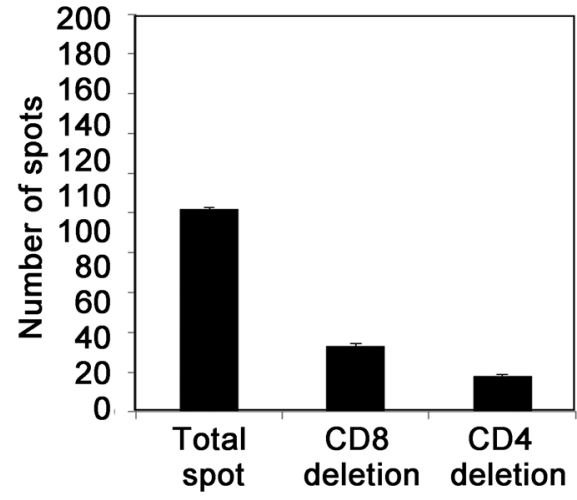

(c)

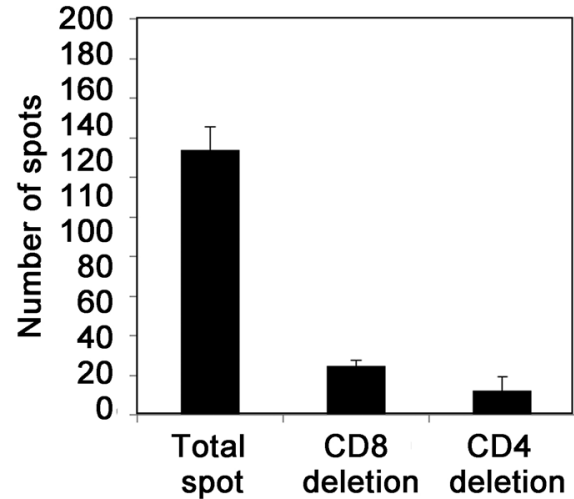

Fig. 6

Norovirus-specific IFN- $\gamma$ response after the intrabuccal administration

Splenocytes from immunized or naïve mice were stimulated with rNoV VLPs, and analyzed for IFN- $\gamma$ production with ELISPOT. Number of spots = antibody-forming cells in $10^{6}$ cells.

\section{Cross reactivity to other genotypes of NoV VLPs}

Fecal immune responses induced by NoV GII.4 VLPs were examined for the cross reactivity with other NoV genotypes including VLPs of NoV GI.1, GI.11, GII.2, and GII.3. It was found that fecal samples at 49 dpi after 2-dose immunization cross-reacted with other genotypes of $\mathrm{NoV}$ VLPs (Fig. 5).

ELISPOT assay for kinetics of IFN- $\gamma$ positive cell response after boost challenge by the intrabuccal administration

We examined the types and proportions of the IFN- $\gamma$ positive cells in splenocyte suspensions from mice that were intrabuccally inoculated once or twice with NoV GII.4 VLPs. The cells were assessed by IFN- $\gamma$ positive ELISPOT assay. It was found that the number of induced IFN- $\gamma$ secreting splenocytes increased after 14 dpi by NoV GII.4 VLPs (Fig. 6a). The levels of CD4 positive T cells or CD8 positive T cells were also examined in the immunized mice. At 14 and $35 \mathrm{dpi}$, splenocytes were depleted with magnetic beads against CD4 positive cells and CD8 positive cells. Mainly CD4 positive cells were decreased (Fig. 6b,c).

\section{Discussion}

Many papers reported that NoV VLPs are an effective immunogen (Atmar et al., 2011; Ball et al., 1998; LoBue et al., 2006; Guerrero et al., 2001; Tacket et al., 2003). We first demonstrated that NoV GII.4 VLPs challenged by the subcutaneous route induced strong serum IgG responses in mice, but failed to induce fecal IgA responses. The development of a vaccine against mucosal infections that can induce secretory IgA had been reported mainly through the oral route (Atmar et al., 2011; Holmgren and Svennerholm, 2012; Tacket et al., 2003). Our present study demonstrated, that titers of serum and fecal IgA after using an intubation needle for oral immunization (gavage injection) in mice without adjuvant which significantly increased, were consist- 
ent with the previous reports (Ball et al., 1998; Periwal et al., 2003). Interestingly, the intrabuccal administration induced both serum and fecal IgA, and serum IgG. In addition, the intrabuccal administration increased antibody titers in the boost trial, especially in the IgG titer. From our results, the intrabuccal VLPs-absorbing route might be in CD4+ T cells dependent manner. In live oral norovirus vaccine studies in mouse model, CD4 T cells, CD8 T cells, and B cells showed broad spectrum of immune responses (Cerutti, 2008). We have showed that NoV VLPs are effective immunogen for IgG, IgA, and CD4+ $\mathrm{T}$ cells induction, even if they are not in form of live vaccine. The IgA induced by NoV GII.4 VLPs have shown to cross react with VLPs of other genotypes, especially the GII.3 genotype. Our data support the previous study about heterologous immune responses to norovirus shown in mice (LoBue et al., 2010). Although neutralizing antibodies could not be defined due to an unavailability of any effective animal model or cell culture system, our results demonstrated that norovirus VLPs were able to enhance immune responses in mice and could be used as a candidate vaccine in humans.

Acknowledgement. This research was supported by a Grant-in-Aid from Japan Society for the Promotion Science (JSPS) - Grant Nos. 25670479 and 24390266.

\section{References}

Atmar RL, Berstein DI, Harro CD, Al-lbrahim MS, Chen WH, Ferreira J, Estes MK, Graham DY, Opekun AR, Richardson C, Mendelman PM (2011): Norovirus vaccine against experimental human Norwalk virus illness. N. Engl. J. Med. 365, 2178-2187. https:/doi.org/10.1056/ NEJMoa1101245

Ball JM, Hardy ME, Atmar RL, Conner ME, Estes MK (1998): Oral immunization with recombinant Norwalk virus-like particles induces a systemic and mucosal immune response in mice. J. Virol. 72, 1345-1353.

Bok K, Green KY (2012): Norovirus gastroenteritis in immunocompromised patients. N. Engl. J. Med. 367, 2126-2132. https:/doi.org/10.1056/NEJMra1207742

Cerutti A (2008): The regulation of IgA class switching. Nat. Rev. Immunol. 8, 286-294. https:/doi.org/10.1038/ $\underline{\text { nri2322 }}$

Fankhauser RL, Monroe SS, Noel JS, Humphrey CD, Bresee JS, Parashar UD, Ando T, Glass RI (2002): Epidemiologic and molecular trends of "Norwalk-like viruses" associated with outbreaks of gastroenteritis in the United States. J. Infect. Dis. 186, 1-7. https:/doi.org/10.1086/341085

Gray JJ, Cunliffe C, Ball J, Graham DY, Desselberger U, Estes MK (1994): Detection of immunoglobulin M (IgM), IgA, and IgG Norwalk virus-specific antibodies by indirect enzyme-linked immunosorbent assay with baculovirusexpressed Norwalk virus capsid antigen in adult volun- teers challenged with Norwalk virus. J. Clin. Microbiol. 32, 3059-3063.

Glass RI, Noel J, Ando T, Fankhauser R, Belliot G, Mounts A, Parashar UD, Bresee JS, Monroe SS (2000): The epidemiology of enteric caliciviruses from humans: A reassessment using new diagnostics. J. Infect. Dis. 181, 254-261. https:/ doi.org/10.1086/315588

Glass RI, Parashar UD, Estes MK (2009): Norovirus gastroenteritis. N. Engl. J. Med. 361, 1776-1785. https:/doi.org/10.1056/ NEJMra0804575

Guerrero RA, Ball JM, Krater SS, Pacheco SE, Clements JD, Estes MK (2001): Recombinant Norwalk virus-like particles administered intranasally to mice induce systemic and mucosal (fecal and vaginal) immune responses. J. Virol. 75, 97139722. https:/doi.org/10.1128/JVI.75.20.9713-9722.2001

Holmgren J, Svennerholm AM (2012): Vaccines against mucosal infections. Curr. Opin. Immunol. 24, 343-353. https:/doi. org/10.1016/j.coi.2012.03.014

Jiang X, Wang M, Graham DY, Estes MK (1992): Expression, self-assembly, and antigenicity of Norwalk virus capsid protein. J. Virol. 66, 6527-6532.

Kroneman A, Vega E, Vennema H, Vinjé J, White PA, HansmanG, GreenK, Martella V, Katayama K, Koopmans M (2013): Proposal for a unified norovirus nomenclature and genotyping. Arch. Virol. 158, 2059-2068. https:/doi. org/10.1007/s00705-013-1708-5

Lindesmith LC, Beltramello M, Donaldson EF, Corti D, Swanstrom J, Debbink K, Lanzavecchia A, Baric RS (2012): Immunogenetic mechanisms driving norovirus GII.4 antigenic variation. PLoS. Pathog. 8, 1-9. https:/doi.org/10.1371/ journal.ppat.1002705

LoBue AD, Lindesmith LC, Baric RS (2010): Identification of cross-reactive norovirus CD4+ T cell epitopes. J. Virol. 84, 8530-8538. https:/doi.org/10.1128/JVI.00727-10

LoBue AD, Lindesmith L, Yount B, Harrington PR, Thompson JM, Johnston RE, Moe CL, Baric RS (2006): Multivalent norovirus vaccines induce strong mucosal and systemic blocking antibodies against multiple strains. Vaccine 24, 52205234. https:/doi.org/10.1016/j.vaccine.2006.03.080

Okamae M, Shiota T, Hansman GS, Takagi M, Yagyu F, Takanashi S, Phan TG, Shimizu Y, Kohno H, Okitsu S, Ushijima H (2007): Anti-Norovirus polyclonal antibody and its potential for development of an antigen-ELISA. J. Med. Virol. 79, 1180-1186. https:/doi.org/10.1002/jmv.20906

Parker SP, Cubitt WD, Jiang X (1995): Enzyme immunoassay using baculovirus-expressed human calicivirus (Mexico) for the measurement of IgG responses and determining its seroprevalence in London, UK. J. Med. Virol. 46, 194-200. https:/doi.org/10.1002/jmv.1890460305

Parker SP, Cubitt WD, Jiang XJ, Estes MK (1994): Seroprevalence studies using a recombinant Norwalk virus protein enzyme immunoassay. J. Med. Virol. 42, 146-150. https:/ doi.org/10.1002/jmv.1890420209

Prasad BV, Hardy ME, Dokland T, Bella J, Rossmann MG, Estes MK (1999): X-ray crystallographic structure of the Norwalk virus capsid. Science 286, 287-290. https:/doi. org/10.1126/science.286.5438.287 
Periwal SB, Kourie KR, Ramachandaran N, Blakeney SJ, DeBruin S, Zhu D, Zamb TJ, Smith L, Udem S, Eldridge JH, Shroff KE, Reilly PA (2003): A modified cholera holotoxin CT-E29H enhances systemic and mucosal immune responses to recombinant Norwalk virus-virus like particle vaccine. Vaccine 21,376-385. https:/doi.org/10.1016/S0264-410X(02)00618-7 Rockx B, Baric RS, de Grijs I, Duizer E, Koopmans MP (2005): Characterization of the homo- and heterotypic immune responses after natural norovirus infection. J. Med. Virol. 77, 439-446. https:/doi.org/10.1002/jmv.20473

Tacket CO, Sztein MB, Losonsky GA, Wasserman SS, Estes MK (2003): Humoral, mucosal, and cellular immune responses to oral Norwalk virus-like particles in volunteers. Clin. Immunol. 108, 241-247. https:/doi.org/10.1016/ $\underline{\mathrm{S} 1521-6616(03) 00120-7}$ 